\title{
MPA influences tumor cell proliferation, migration, and invasion induced by RANKL through PRB involving the MAPK pathway in endometrial cancer
}

\author{
JING WANG $^{1^{*}}$, XIAO SUN ${ }^{2 *}$, HUIJUAN ZHANG ${ }^{3}$, YUDONG WANG $^{1}$ and YUHONG LI ${ }^{1}$ \\ ${ }^{1}$ Department of Gynecology, ${ }^{2}$ Laboratory for Gynecologic Oncology, and \\ ${ }^{3}$ Department of Pathology and Biobank, International Peace Maternity and Child Health Hospital \\ Affiliated to Shanghai Jiao Tong University School of Medicine, Shanghai 200030, P.R. China
}

Received August 29, 2014; Accepted October 30, 2014

DOI: 10.3892/or.2014.3651

\begin{abstract}
The targeting of receptor activator of nuclear factor- $\kappa \mathrm{B}$ ligand (RANKL) is being increasingly investigated as a potential therapeutic strategy in several types of cancers. However, the exact function and mechanism of RANKL in human endometrial cancer (EC), particularly in progesteroneresistant and aggressive EC, remain unclear. We evaluated whether targeting of RANKL might be an efficient therapeutic strategy in EC. In the present study, we performed the first investigation of the relationship between RANK/RANKL expression in EC tissues and clinicopathological features. In the present study, we showed that RANK/RANKL was aberrantly overexpressed in human EC tissues. The higher RANK expression in human EC was associated with myometrial invasion, lymph node metastasis and lymphovascular space involvement. Additionally, we discovered that RANK/RANKL promoted EC cell proliferation, migration and invasion, which was correlated with the activated mitogen-activated protein kinase (MAPK) pathway. Moreover, medroxyprogesterone acetate (MPA)-mediated progesterone receptor B (PRB) was found to significantly inhibit the EC cell behavior induced by RANKL in vitro. Furthermore, MPA efficiently inhibited the tumorigenicity in an in vivo xenograft model. Collectively, RANKL is a common tumor promoter, which activates MAPK signaling in EC cells. MPA-mediated PRB plays important
\end{abstract}

Correspondence to: Dr Yudong Wang or Dr Yuhong Li, Department of Gynecology, International Peace Maternity and Child Health Hospital Affiliated to Shanghai Jiao Tong University School of Medicine, Shanghai 200030, P.R. China

E-mail: owangyudong@126.com

E-mail: liyuhong101@msn.com

*Contributed equally

Key words: receptor activator of nuclear factor- $\mathrm{\kappa B}$, receptor activator of nuclear factor- $\kappa \mathrm{B}$ ligand, endometrial cancer, MPA, progesterone receptor $\mathrm{B}$ roles in inhibiting the growth, migratory and invasive capacities of EC cells induced by RANKL. Targeting of RANKL may be useful in the treatment of EC.

\section{Introduction}

Endometrial cancer (EC) is one of the most common gynecological malignant tumors. In the United States, 52,630 new cases of EC will be diagnosed and 8,590 deaths are projected in 2014 (1). Although EC is efficiently diagnosed and successfully treated, the treatment of progesterone-resistant and aggressive EC is difficult, and is associated with decreased survival rates $(2,3)$. Women with variants of endometrioid adenocarcinoma, high-grade histology, deep myometrial invasion, a tumor that invades the uterine cervix, or a tumor that spreads to a local or regional site have high risks of metastasis and recurrence. Additionally, lower levels of progesterone receptor $\mathrm{B}(\mathrm{PRB})$ are positively correlated with progesterone resistance and poor prognosis. Therefore, reasonable and effective treatment can significantly improve the survival of patients with progesterone-resistant and aggressive EC. However, the molecular mechanisms underlying EC are not yet fully elucidated.

A novel cytokine system that promotes tumor metastasis has been identified and is composed of the receptor activator of nuclear factor- $\kappa \mathrm{B}$ (RANK), its ligand RANKL and osteoprotegerin (OPG). RANKL, a member of the tumor necrosis factor (TNF) receptor superfamily, is expressed primarily in lymphoid tissues and in osteoblastic cell lines (4). RANK is essential for the development of lactating mammary glands $(5,6)$ and the formation of lymph nodes. Recent reports suggest that the binding of RANKL to RANK triggers a host of intracellular reactions through a TNF-related molecule signaling pathway, which promotes the metastatic behavior of malignant epithelial cells (7-10). It has been demonstrated that RANK/RANKL is associated with the initiation of tumorigenesis, and the progression and invasion of tumors in human mammary epithelial cells (11-13). In addition, this system also promotes progestin-driven primary mammary cancer development under the control of sex hormones $(11,12,14)$. OPG, a decoy receptor, blocks the binding of RANKL to RANK 
(thus preventing RANK activation), which is a secreted protein lacking transmembrane domains.

It has been verified that medroxyprogesterone acetate (MPA) can induce the expression of RANKL in the mammary gland (12), which results in an acceleration of tumorigenesis after multiparity or treatment with progesterone and carcinogens (11). Moreover, recent research has demonstrated that the RANK/RANKL pathway is involved in the incidence of progesterone-driven breast cancer. MPA has been identified as an efficacious and well-tolerated treatment for EC for several decades. Intriguingly, we found that RANK/RANKL was expressed in EC at levels similar to those found in $\mathrm{BC}$. The interaction of PRB/MPA and RANK/RANKL is poorly understood in EC and warrants further elucidated.

In the present study, we performed the first investigation of the relationship between RANK/RANKL expression in EC tissues and clinicopathological features and revealed that RANK/RANKL expression was markedly higher in malignant tumors than that in normal endometrium. Then, we examined the effect of MPA on cell behavior induced by RANKL in different EC cell lines (Ishikawa and KLE), and we determined whether the PRB is associated with progesterone resistance. We demonstrated that the RANK-RANKL system can play a pivotal role in EC progression via the mitogen-activated protein kinase (MAPK) pathway, which can be abrogated by the effect of MPA-mediated PRB in Ishikawa cell. Given the promising results, targeting of RANKL might serve as an appropriate therapy for EC, particularly progesterone-resistant or aggressive EC.

\section{Materials and methods}

Tissue selection and patient characteristics. Paraffinembedded endometrial tissue samples were collected from May 2011 to March 2013. Seventy tissue samples of EC were obtained from patients, who underwent surgical staging and tumor grading of the disease established in accordance with the criteria of the Federation International of Gynecology and Obstetrics (FIGO) staging system. Thirty normal endometrial tissues were collected from patients who underwent hysterectomies due to myoma, adenomyosis or other diseases. Twenty endometrial atypical hyperplasia (EAH) samples were also selected from patients who underwent hysteroscopy due to irregular vaginal bleeding. Twenty BC samples were collected from patients following adenomammectomy. None of the patients involved in the present study received hormonotherapy or adjuvant radiation therapy before surgery.

Reagents and antibodies. Recombinant human RANK ligand was obtained from PeproTech (Rocky Hill, NJ, USA). MPA was purchased from Sigma. Rabbit anti-phospho-ERK1/2, -ERK1/2, -phospho-JNK, -JNK, -phospho-p38, -p38, -RANK, -RANKL, -PRB, -NF- $\mathrm{B} 2$, -H3, -Ki-67, -PARP and -caspase-3 antibodies were purchased from Cell Signaling Technology.

Immunohistochemical analysis. The expression levels of RANK and RANKL were detected through the avidin-biotin complex/immunoperoxidase method. Endometrial tissue samples were sliced into $4-\mu \mathrm{m}$-thick tissue sections. The deparaffinized sections were then boiled in 1:50 ethylene diamine tetraacetic acid for antigen retrieval. The specimens were incubated with $3 \%$ hydrogen peroxide. After blocking with serum, the sections were incubated with primary antibodies against RANK and RANKL. The antibody binding was detected using reagents (Miao Tong, Shanghai, China). Two independent investigators without knowledge of the clinical pathological parameters evaluated the expression of RANK/RANKL. The assessment method was as follows. Based on the staining intensity, the sections were rated as negative (0), weak (1), medium (2), or strong (3). In addition, combined with the extent of staining, the sections were scored as $0(0 \%), 1(1-25 \%), 2(26-50 \%), 3(51-75 \%)$ or $4(76-100 \%)$. The sum of the intensity and extent of staining was considered the final staining value of the paraffin sections. A value of at least 4 was considered to indicate positive expression.

Cell cultures. Human EC cell lines (Ishikawa and KLE) were purchased from the American Type Culture Collection (ATCC, Manassas, VA, USA). The cells were cultured in Dulbecco's modified Eagle's medium-F12 (Gibco, Auckland, New Zealand) supplemented with $10 \%$ fetal bovine serum (FBS; Gibco, Carlsbad, CA, USA), $100 \mu \mathrm{g} / \mathrm{ml}$ streptomycin, and $100 \mathrm{U} / \mathrm{ml}$ penicillin (HyClone) at $37^{\circ} \mathrm{C}$ in a humidified atmosphere of $5 \% \mathrm{CO}_{2}$.

Vector construction and lentiviral transduction. The human RANK gene (NM_001270949.1) was cloned into pL-TO-IRESLUC to construct a lentiviral vector overexpressing the RANK gene (Nuo Bai, Shanghai, China). Ishikawa and KLE cells were transduced with the viral supernatant of pL-TO-IRESLUC (empty vector, EV) and pL-TO-IRES-LUC-RANK, respectively, in the presence of $8 \mu \mathrm{g} / \mathrm{ml}$ Polybrene. Stable cell lines were selected using BSD (1.5 and $2 \mu \mathrm{g} / \mathrm{ml})$. The clones were confirmed through quantitative real-time polymerase chain reaction (qRT-PCR) and western blot analysis.

Co-administration of MPA and RANKL. A total of $1 \times 10^{6}$ Ishikawa and $3 \times 10^{6}$ KLE cells were plated separately in 6-well plates. The cells were treated with MPA, RANKL, MPA + RANKL, and an equal volume of double-distilled water (control). After the treatments, the proteins were extracted and detected by western blot analysis.

Cell proliferation assay. The cell proliferation was assessed using a standard 3-(4,5-dimethylthiazol-2-yl)-2,5-diphenyltetrazolium bromide (MTT) (Sigma, St. Louis, MO, USA) assay. The absorbance values were calculated at $490 \mathrm{~nm}$ using a SpectraMax 190 microplate reader (Bio-Rad Model 680).

Migration and invasion assays. A total of $1 \times 10^{5}$ cells were seeded into the upper chamber of a 24-well chemotaxis chamber with polycarbonate filters $(8-\mu \mathrm{m}$ pore) (Corning Incorporated, Glendale, AZ, USA). The cells were treated with 1,2 and $2.5 \mu \mathrm{g} / \mathrm{ml}$ RANKL for $4 \mathrm{~h}$. The crystal violet-stained cells in five fields/well were counted at x200 magnification. The invasion of cells was performed using Transwell chambers with $8-\mu \mathrm{m}$ pore membranes coated with $40 \mu \mathrm{l}$ of Matrigel at a 1:6 dilution (BD Biosciences, San Jose CA, USA) on the upper side. A total of $1 \times 10^{5}$ cells in $100 \mu 1$ of serum-free medium and $500 \mu \mathrm{l}$ of complete medium were added to the upper chamber 
and lower chamber, respectively. After incubation for 12, 24 and $48 \mathrm{~h}$ separately, the cells were fixed with $4 \%$ paraformaldehyde and counted as described above.

Apoptosis analysis. MPA $(10 \mu \mathrm{M}), \mathrm{RANKL}(1 \mu \mathrm{g} / \mathrm{ml}), \mathrm{MPA}+$ RANKL, or an equal volume of dimethyl sulfoxide (control) were added to the cells, and the plates were incubated for $48 \mathrm{~h}$. The cells were digested, washed and resuspended in binding buffer. Annexin V-FITC and PI were then added (BD Pharmingen, San Diego, CA, USA).

RNA extraction and real-time PCR analysis. Total RNA was extracted with TRI reagent (Invitrogen, Shanghai, China) and the quality of the total RNA was assessed using a spectrophotometer (Pharmacia Biotech RNA/DNA calculator). The RNA $(500 \mathrm{ng})$ was reverse transcribed into cDNA using a reverse transcription kit (Takara, Dalian, China). qRT-PCR was performed with SYBR-Green Master Mix (Takara) on an ABI Prism 700 thermal cycler (Applied Biosystems, Foster City, CA, USA) to detect the expression of various genes. The target gene expression was calculated using the $\Delta \Delta \mathrm{Ct}$ method with $\beta$-actin as the housekeeping gene. The following primers were used: $\beta$-actin, 5 '-CAGCCATGTACGTTGCTATCCAGG-3' and 5'-AGGTCCAGACGCAGGATGGCATG-3'; PRB, 5'-TGCCCAGCATGTCGCCTTAG-3' and 5'-CTGGCTTAG GGCTTGGCTTTC-3'; RANK, 5'-AGCATTATGAGCATC TGGGACGG-3' and 5'-CAGCAAGCATTTATCTTCTTCA TTCC-3'.

Western blot analysis. For total protein extraction, the cells were harvested and lysed with RIPA buffer containing protease and phosphatase inhibitor cocktails. The nuclear protein was extracted using NE-PER nuclear and cytoplasmic extraction reagents according to the manufacturer's protocol. We used the following antibodies: ERK1/2, p-ERK1/2, JNK, p-JNK, p38, p-p38, NF-кB2 (p52, p100), H3, caspase-3 and PARP. $\beta$-actin expression was used as the control.

Enzyme-linked immunosorbent assay (ELISA). OPG and RANKL serum concentrations were measured using commercially available immunoenzymatic assay kits, respectively, according to the manufacturer's instructions (Ray Biotech, Norcross, GA, USA; Abnova, Taipei, Taiwan). The ELISA kit is an in vitro enzyme-linked immunosorbent assay for quantitative measurement. A monoclonal antibody against the mouse was employed as capture antibody and a biotinylated detection polyclonal antibody from goat was used as the detection antibody.

Nude mouse tumor xenograft assay. Twenty 6-week-old female nude BALB/c mice were obtained from the Shanghai Life Science Institute (Slac Laboratory Animal Co., Ltd., Shanghai, China). Animal research was carried out in strict accordance with the recommendations in the Guideline for the Care and Use of Laboratory Animals of China. The procedures were approved by the Committee on the Ethics of Animal Experiments of the Obstetrical and Gynecological Hospital affiliated Fudan University [Permit Number: SYXK (hu) 20080064]. All efforts were made to minimize animal suffering. In order to investigate the interaction between MPA and RANKL in regards to tumor growth in vivo, Ishikawa cells transfected with the lentiviral vector carrying the human RANK gene were used. All mice were randomly divided into four groups of five mice to receive $1 \times 10^{7}$ cells, and were treated with either vehicle (control), MPA (100 mg/kg body weight), RANKL (250 $\mu \mathrm{g} /$ $\mathrm{kg}$ ) or MPA + RANKL, respectively. The cells were injected subcutaneously into the groin of each mouse. Subcutaneous tumor formation was monitored one week after injection and was measured weekly using digital calipers. The tumors were removed after 28 days. Tumor volume $\left(\mathrm{mm}^{3}\right)$ was calculated using the following formula: Tumor volume $\left(\mathrm{mm}^{3}\right)=$ (the longest diameter) $\mathrm{x}$ (the shortest diameter) ${ }^{2} \mathrm{x} 0.5$.

Statistical analysis. The data were analyzed using the SPSS 17.0 software (SPSS, Inc., Chicago, IL, USA). The statistical analyses were performed using an unpaired Student's t-test or one-way ANOVA. The Chi-square test was employed to compare categorical data. All of the experiments were performed in triplicate. $\mathrm{P}<0.05$ was considered statistically significant. All data are presented as means with standard deviations (SD)

\section{Results}

RANK/RANKL is upregulated in EC and is correlated with clinicopathological parameters. To determine the effect of RANK/RANKL expression on the development and progression of EC, we performed immunohistochemistry (IHC) on the endometrial tissues. The RANK/RANKL staining was predominantly localized to the cytoplasmic membrane of the endometrial epithelial cells. Weak or no staining was observed in the normal endometrium, and moderate to strong RANK/RANKL staining was found in endometrial atypical hyperplasia (EAH), endometrial adenocarcinoma (EA), uterine papillary serous carcinoma (UPSC) and breast cancer (BC) (Fig. 1A). Briefly, most normal endometrium samples were negative for RANK/RANKL (mean IHC value $\leq 2)$, and most of the EAH samples exhibited weak staining (mean IHC value <3), whereas the majority of the EC tissues presented positive expression of RANK/RANKL (mean IHC value $>4$ ) (Fig. 1B). We explored the correlation between RANK/RANKL expression and the clinicopathological characteristics of EC. Higher RANK/RANKL expression levels were observed in carcinomas with myometrial invasion $(\mathrm{P}=0.006$; Table I), lymph node metastasis $(\mathrm{P}=0.045$; Table I) and lymphovascular space involvement $(\mathrm{P}=0.025$; Table I). We did not, however, find any correlations between RANK/RANKL and other clinicopathological characteristics, such as age, FIGO stage, histological type, pathological grade or expression of estrogen receptor (ER)/progesterone receptor (PR) $(\mathrm{P}>0.05$; Table I). This result indicated that the expression levels of RANK/RANKL play a pivotal role in the risk-associated clinicopathological characteristics of EC. RANK/RANKL overexpression increased the RANKL secretion in EC patients, whereas the serum level of OPG was lower than that in the healthy controls (Fig. $1 \mathrm{C}$ and D).

Determination of PRB expression in five EC cell lines and verification of RANK overexpression. To confirm the endogenous PRB expression in EC cell lines (Ishikawa, HEC-1B, AN3CA, 
A

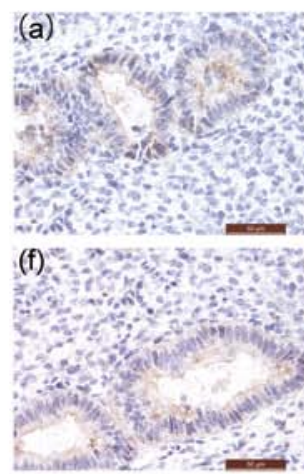

Normal
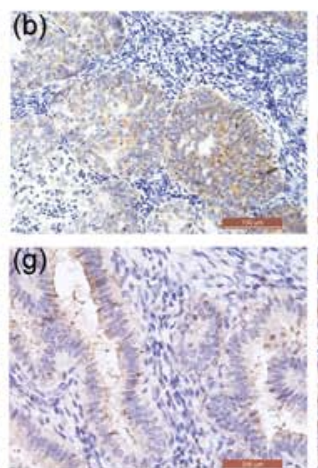

Endometrioid
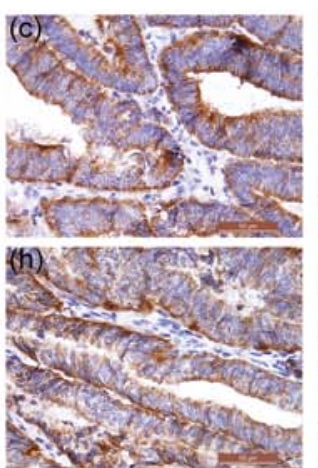

Endometrioid

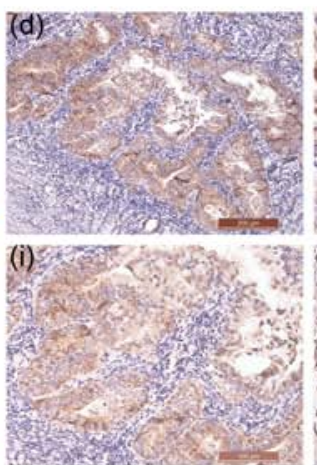

Uterine Papillary
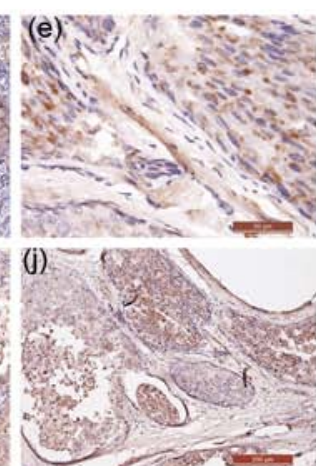

Breast

Endometrium Atypical Hyperplasia Adenocarcinoma Serous Carcinoma Cancer

B

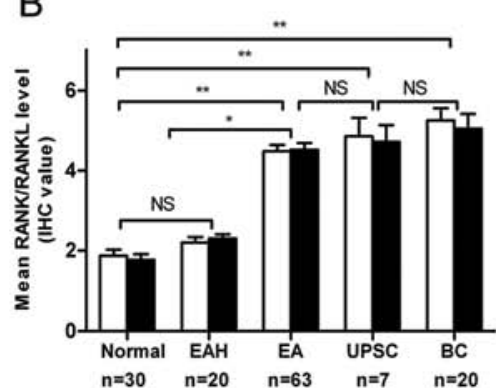

C $\square$ RANK

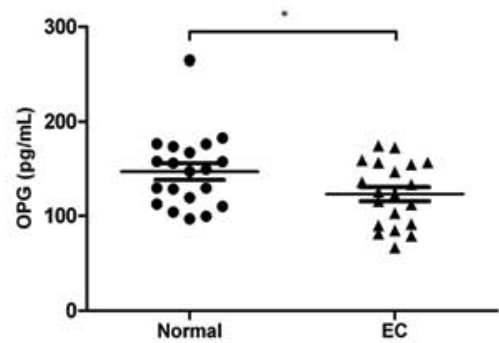

D

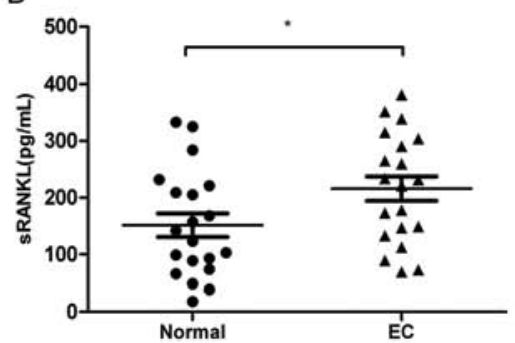

Figure 1. RANK/RANKL expression in endometrium tissue specimens and the serum levels of OPG and RANKL. (A) Immunohistochemical analysis of RANK and RANKL expression in normal endometrium, endometrial atypical hyperplasia (EAH), endometrial adenocarcinoma (EA), uterine papillary serous carcinoma (UPSC) and breast cancer (BC). Scale bar $=100 \mu \mathrm{m}$. (B) Statistical results of the immunostaining values. (C and D) The serum levels of OPG and RANKL. "P $<0.05 ;{ }^{* *} \mathrm{P}<0.01 ; \mathrm{NS}$, not significant.

A

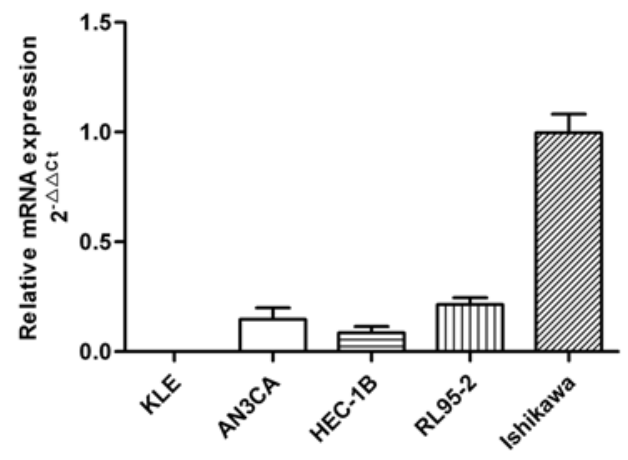

B

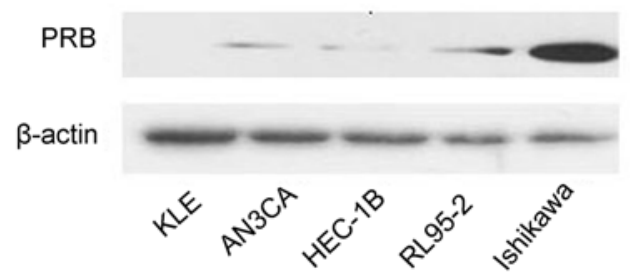

C

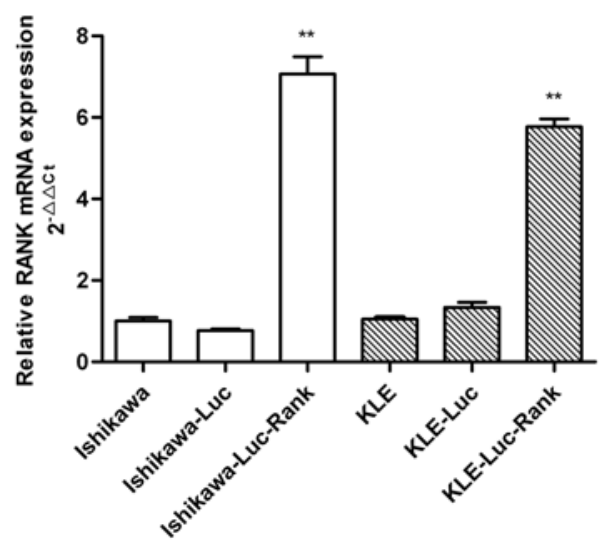

$\mathrm{D}$

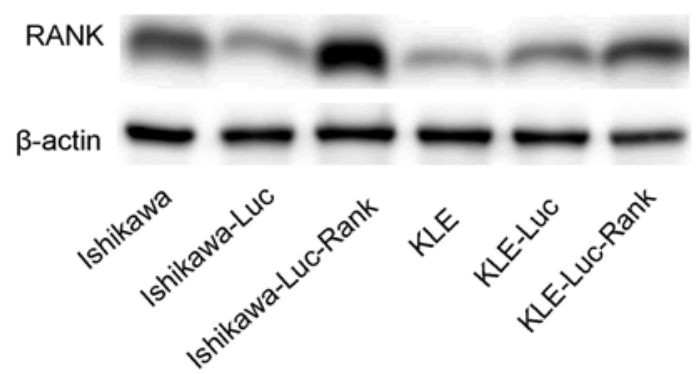

Figure 2. Determination of PRB expression in five EC cell lines and verification of the overexpression of RANK. (A) PRB expression was analyzed by qRT-PCR. (B) The protein lysates from different EC cell lines were measured for PRB expression by western blot analysis. $\beta$-actin was used as the loading control. (C) qRT-PCR and (D) western blot analysis were also employed to validate the overexpression of the RANK gene in the Ishikawa-Luc-Rank and KLE-Luc-Rank cells. 
Table I. Correlation between RANK/RANKL expression and clinicopathological characteristics of the endometrial cancer cases.

\begin{tabular}{|c|c|c|c|c|c|c|c|c|}
\hline \multirow[b]{2}{*}{ Parameters } & \multirow[b]{2}{*}{ Patients (n) } & \multirow[b]{2}{*}{ Patients (\%) } & \multicolumn{3}{|c|}{ RANK expression } & \multicolumn{3}{|c|}{ RANKL expression } \\
\hline & & & Negative & Positive & P-value & Negative & Positive & P-value \\
\hline Total & 70 & 100 & 13 & 57 & & 12 & 58 & \\
\hline Age (years) & & & & & 0.452 & & & 0.088 \\
\hline$<50$ & 16 & 22.9 & 4 & 12 & & 5 & 11 & \\
\hline$\geq 50$ & 54 & 77.1 & 9 & 45 & & 7 & 47 & \\
\hline \multicolumn{9}{|c|}{ Grade (endometrioid=63) } \\
\hline $\mathrm{G} 1$ or $\mathrm{G} 2$ & 57 & 90.5 & 8 & 49 & 0.219 & 9 & 48 & 0.955 \\
\hline G3 & 6 & 9.5 & 2 & 4 & & 1 & 5 & \\
\hline \multicolumn{9}{|l|}{ FIGO stage } \\
\hline I or II & 67 & 95.7 & 12 & 55 & 0.502 & 11 & 56 & 0.447 \\
\hline III or IV & 3 & 4.3 & 1 & 2 & & 1 & 2 & \\
\hline \multicolumn{9}{|l|}{ Histological type } \\
\hline Endometrioid & 63 & 90 & 10 & 53 & 0.082 & 10 & 53 & 0.398 \\
\hline Non-endometrioid & 7 & 10 & 3 & 4 & & 2 & 5 & \\
\hline \multicolumn{9}{|l|}{ Myometrial invasion } \\
\hline$<1 / 2$ & 49 & 70 & 5 & 44 & $0.006^{\mathrm{a}}$ & 4 & 45 & $0.002^{\mathrm{a}}$ \\
\hline$\geq 1 / 2$ & 21 & 30 & 8 & 13 & & 8 & 13 & \\
\hline \multicolumn{9}{|l|}{ Positive lymph nodes } \\
\hline No & 42 & 60 & 11 & 31 & $0.045^{\mathrm{a}}$ & 11 & 31 & $0.014^{\mathrm{a}}$ \\
\hline Yes & 28 & 40 & 2 & 26 & & 1 & 27 & \\
\hline \multicolumn{9}{|c|}{ Lymphovascular space involvement } \\
\hline No & 46 & 65.7 & 12 & 34 & $0.025^{\mathrm{a}}$ & 10 & 36 & 0.158 \\
\hline Yes & 24 & 34.3 & 1 & 23 & & 2 & 22 & \\
\hline \multicolumn{9}{|l|}{ ER expression } \\
\hline Negative & 15 & 21.4 & 4 & 11 & 0.363 & 1 & 14 & 0.225 \\
\hline Positive & 55 & 78.6 & 9 & 46 & & 11 & 44 & \\
\hline \multicolumn{9}{|l|}{ PR expression } \\
\hline Negative & 23 & 32.9 & 3 & 20 & 0.405 & 5 & 18 & 0.475 \\
\hline Positive & 47 & 67.1 & 10 & 37 & & 7 & 40 & \\
\hline
\end{tabular}

${ }^{a} \mathrm{P}<0.05$, for the difference between RANK/RANKL expression in patients with or without $\geq 1 / 2$ myometrial invasion, lymph node metastasis or lymphovascular space involvement. RANK, receptor activator of nuclear factor- $\mathrm{\kappa B}$; RANKL, receptor activator of nuclear factor- $\mathrm{kB}$ ligand; ER, estrogen receptor; PR, progesterone receptor.

RL95-2 and KLE), qRT-PCR and western blot analysis were employed to determined the PRB mRNA and protein level. The PRB protein was expressed at a low level in the HEC-1B, AN3CA, RL95-2 cells, particularly in the KLE cells, and at a relatively high level in the Ishikawa cells (Fig. 2A and B). Thus, we chose the Ishikawa and KLE cells for further experiments. To explore the interaction of RANK/RANKL with MPA/PRB, pL-TO-IRES-LUC-RANK was transfected into the Ishikawa and KLE cells to construct stable cell lines that overexpress the RANK gene (Fig. 2C and D). Subsequently, qRT-PCR and western blot analysis were used to verify the upregulation of RANK expression.

MPA influences the cell biological behaviors of EC cells induced by RANKL in vitro. Accumulating evidence suggests the involvement of RANK/RANKL in the regulation of cancer metastasis; thus, we aimed to ascertain whether the upregulation of RANK influences the behavior of Ishikawa and KLE cells. We tested the interaction between MPA and RANKL in the cell viability of the EC cell lines. MTT assays indicated that the MPA-treated cells had exceptionally poor viability, whereas cell viability steadily increased after RANKL treatment. The combination of MPA and RANKL decreased the cell viability compared to RANKL treatment alone (data not shown). We also performed wound healing assays at $12 \mathrm{~h}$ after establishing the wound. MPA-treated Ishikawa-Luc-Rank cells had a reduced wound closure when compared with the control group, whereas RANKL-treated Ishikawa-Luc-Rank cells had a greater extent of wound closure (Fig. 3A and B).

To further investigate cell behaviors, we also assayed the migratory and invasive capacities. There was a marked increase in the migratory capacity of the Ishikawa-Luc-RANK 
A
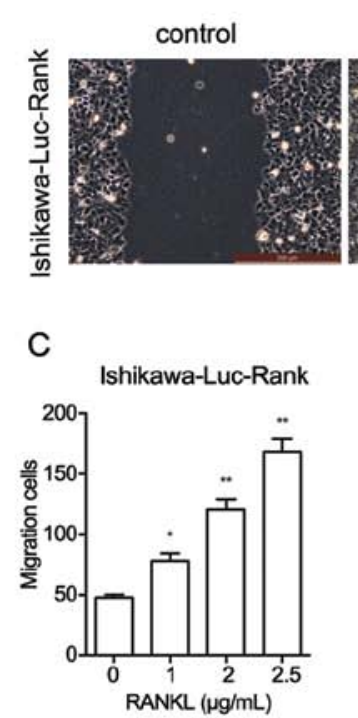
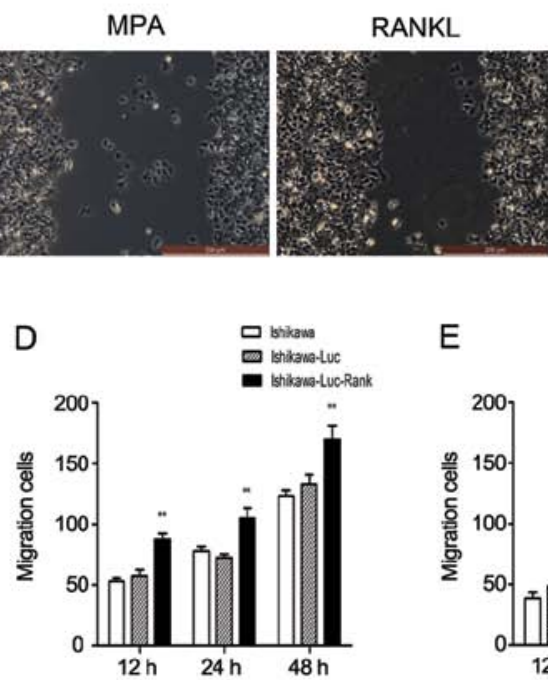

E
B
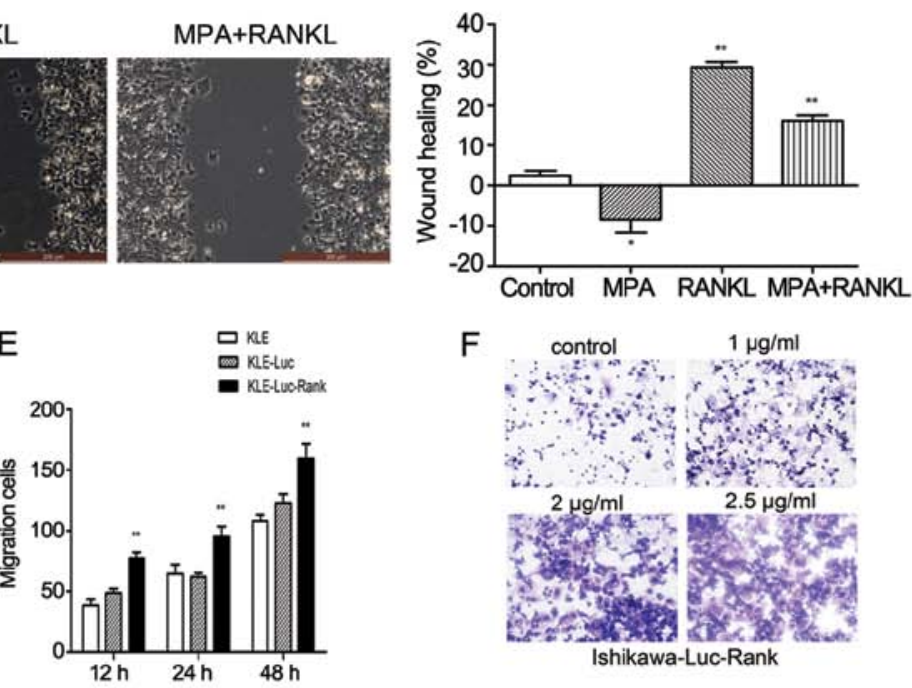

F

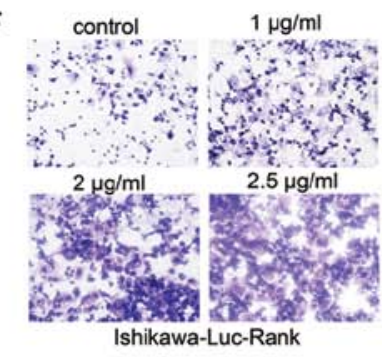

G
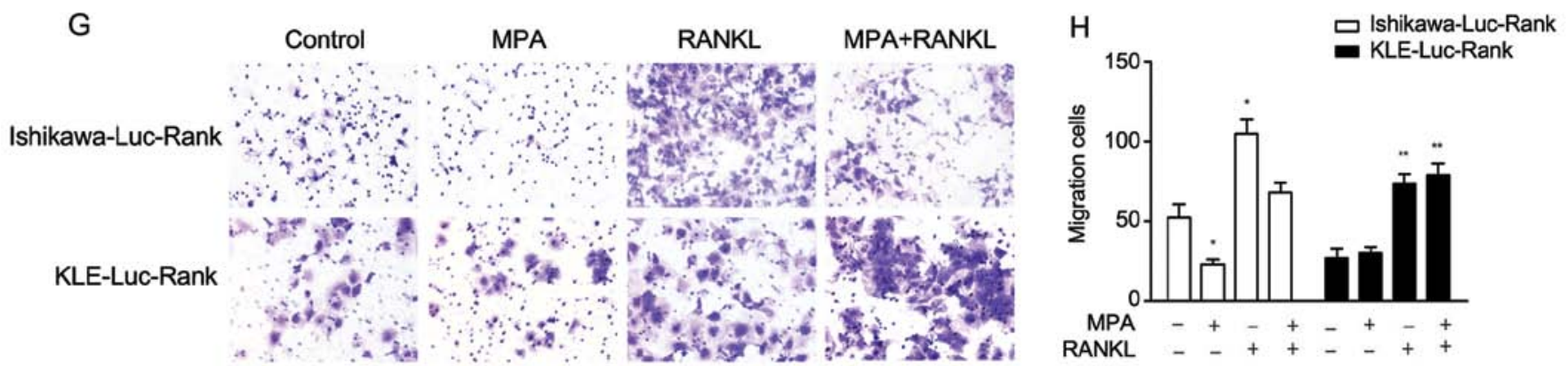

I

Control

MPA

RANKL

MPA+RANKL

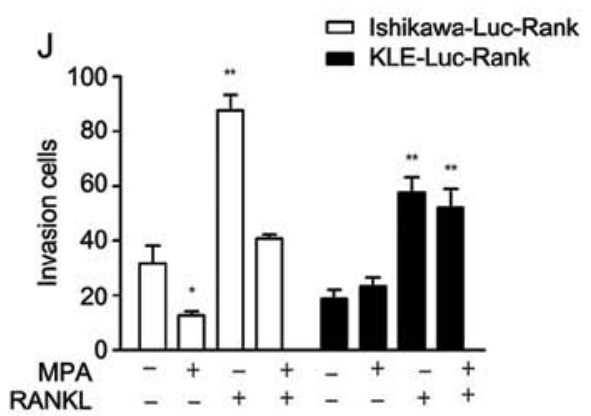

Figure 3. MPA influences the biological behaviors of EC cells induced by RANKL in vitro. (A and B) Wound healing assays to test the migratory capacity following the different treatments. MPA-treated cells demonstrated markedly decreased wound closure, whereas RANKL significantly promoted the wound closure. (C-F) RANKL increased the migration of Ishikawa-Luc-Rank cells in a dose- and time-dependent manner. Ishikawa-Luc-Rank cells showed a clear increase in migratory ability compared to the Ishikawa and Ishikawa-Luc cells stimulated with RANKL (1 $\mu \mathrm{g} / \mathrm{ml})$ and exhibited a similar effect to KLE-LucRank cells during the time period. (G-J) The combination of MPA and RANKL had no effect on inhibiting the migration and invasion of KLE-Luc-Rank cells, whereas it reduced the migratory and invasive numbers of the Ishikawa-Luc-Rank cells. ${ }^{*} \mathrm{P}<0.05 ;{ }^{* *} \mathrm{P}<0.01$.

and KLE-Luc-Rank cells. Moreover, the effect of RANKL on cell migration was dose- and time-dependent (Fig. 3C-F). The increase in cell migration in response to $1 \mu \mathrm{g} / \mathrm{ml}$ RANKL was almost $40 \%$ compared to the control cells in the Ishikawa-Luc-Rank cell line. To further explore the interaction between MPA and RANKL in the migratory and invasive capacities, Ishikawa-Luc-Rank cells and KLE-Luc-Rank cells were treated with MPA, RANKL or MPA + RANKL. MPA obviously inhibited the migration and invasion of the Ishikawa-Luc-Rank cells compared to the untreated cells, whereas no effect was observed in the KLE-Luc-Rank cells. The degree of invasion was markedly increased in response to
$1 \mu \mathrm{g} / \mathrm{ml}$ RANKL in both cell lines. The combination of MPA and RANKL also had no effect on inhibiting the migratory and invasive capacities of KLE-Luc-Rank cells, whereas it reduced the migratory and invasive numbers of Ishikawa-LucRank cells (Fig. 3G-J). These findings collectively indicated that the RANK-RANKL system may contribute to the migration and invasion of EC cells and that MPA/PRB interfered with the role of RANK/RANKL.

Effect of the RANK-RANKL pathway on the apoptosis of EC cells. To elucidate the effects of MPA, RANKL and MPA + RANKL on the apoptosis of these cells after a 48-h treatment, 
A Ishikawa-Luc-Rank control

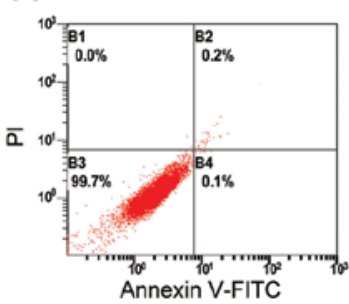

KLE-Luc-Rank control

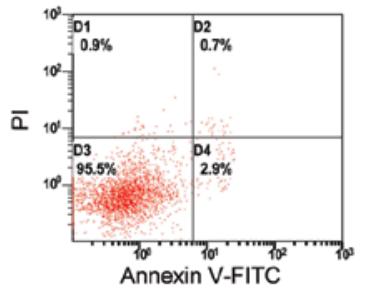

B

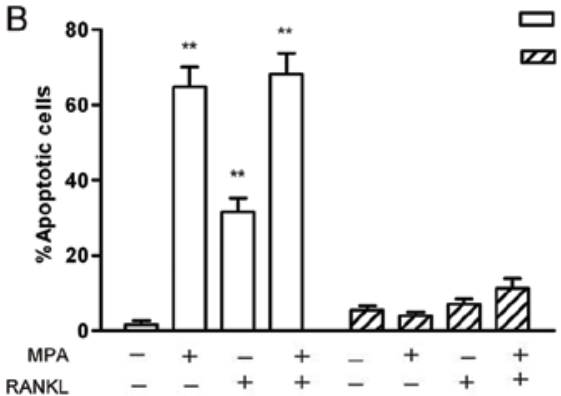

Ishikawa-Luc-Rank MPA

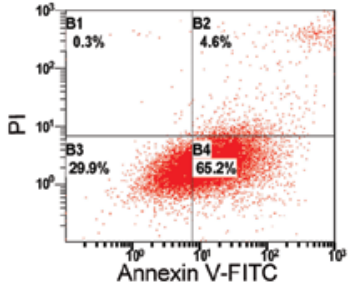

KLE-Luc-Rank MPA

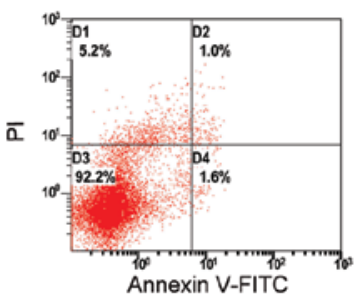

Ishikawa-Luc-Rank KLE-Luc-Rank
Ishikawa-Luc-Rank RANKL

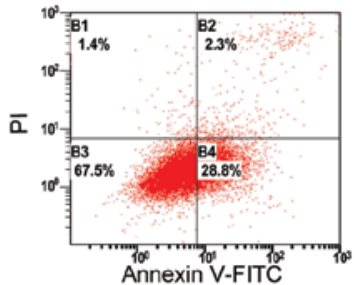

KLE-Luc-Rank RANKL

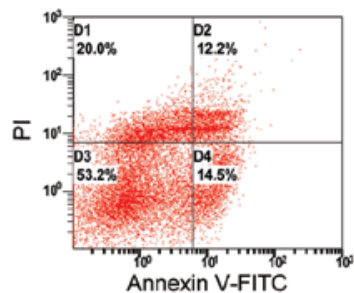

Ishikawa-Luc-Rank MPA+RANKL

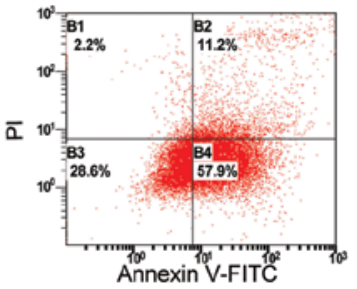

KLE-Luc-Rank MPA+RANKL

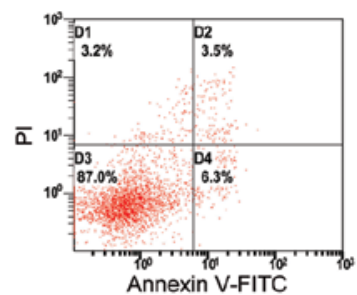

C

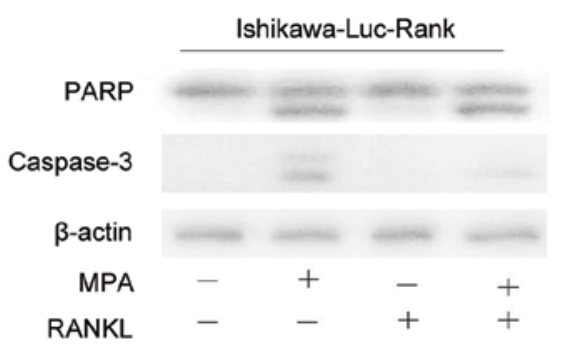

Figure 4. RANK/RANKL has no significant effect on apoptosis. (A and B) The apoptotic cells were detected by flow cytometry. The RANKL stimulus did not obviously affect apoptosis compared with the MPA treatment. RANKL did not promote the apoptosis-inducing effects of MPA in the Ishikawa-Luc-Rank cells. MPA almost had no effect on inducing apoptosis in the KLE-Luc-Rank cells. (C) The protein levels of apoptotic markers were detected by western blot analysis.

flow cytometry and western blot analysis were performed. As demonstrated in Fig. 4, MPA significantly induced the apoptosis of the Ishikawa-Luc-Rank cells. However, the administration of MPA and RANKL had no obvious promoting effects on apoptosis compared to the MPA treatment. Thus, RANKL did not promote the apoptosis-inducing effects of MPA in Ishikawa-Luc-Rank cells. In addition, MPA almost had no effect on inducing apoptosis of the KLE-Luc-Rank cells (Fig. 4A and B).

To further elucidate the effects of RANK/RANKL on apoptosis at the protein level, we detected the antibody levels of caspase-3 and PARP. Consistent with the flow cytometry results, MPA induced apoptosis through activation of caspase-3 and PARP, whereas RANKL did not obviously promote apoptosis (Fig. 4C).

RANK-RANKL system activates the MAPK and $N F-\kappa B$ signaling pathways. To further clarify the mechanisms responsible for the RANK/RANKL regulation of tumor cell behavior, we noted that the interaction between RANK and RANKL resulted in an obvious increase in ERK1/2 and JNK phosphorylation levels in the Ishikawa-Luc-Rank cells. A time-course analysis of the activation of the molecules in the MAPK pathway after treatment with $1 \mu \mathrm{g} / \mathrm{ml}$ RANKL was performed using the relevant antibodies. The results showed that exposure to RANKL induced the phosphorylation of both
ERK1/2 and JNK in the Ishikawa-Luc-Rank and KLE-LucRank cells, whereas there was no significant change in the phosphorylation level of p38 (Fig. 5A). Meanwhile, we also demonstrated the activation of the $\mathrm{NF}-\kappa \mathrm{B}$ pathway, since the activated form of the NF- $\kappa \mathrm{B} 2$ protein (p52) was detected in the cell nuclei (Fig. 5B). The effects of MPA, RANKL and MPA + RANKL on the activation of the MAPK pathway were further examined. The Ishikawa-Luc-Rank cells demonstrated sensitivity to MPA treatment. Conversely, the MPA-treated KLE-Luc-Rank cells did not exhibit the inhibition of the phosphorylation levels of ERK1/2 and JNK. The combination of MPA and RANKL abrogated the activation of ERK1/2 and JNK induced by RANK/RANKL in the Ishikawa-Luc-Rank cells, whereas it had no effect on the KLE-Luc-Rank cells (Fig. 5C and D).

Oncogenic role of RANKL in a tumor xenograft model. To further evaluate the effect of MPA, RANKL and MPA + RANKL on tumorigenesis in vivo, we measured tumor volumes in xenografted mice over a 4-week period. These measurements indicated that in the MPA- and MPA + RANKL-treated groups tumors grew dramatically slower than those in the vehicle- or RANKL-treated groups (Fig. 6A and B). Four weeks after injection, the mice were sacrificed and tumors were removed from the mice. The final mean body weights in the vehicle and RANKL groups were lower than those in the other two groups 


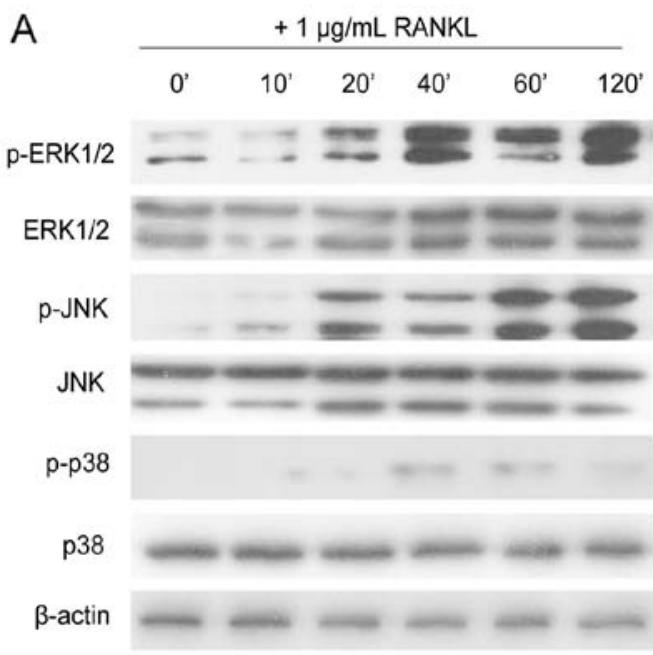

Ishikawa-Luc-Rank

B

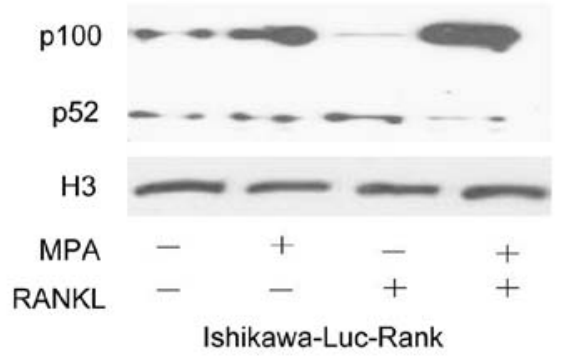

C

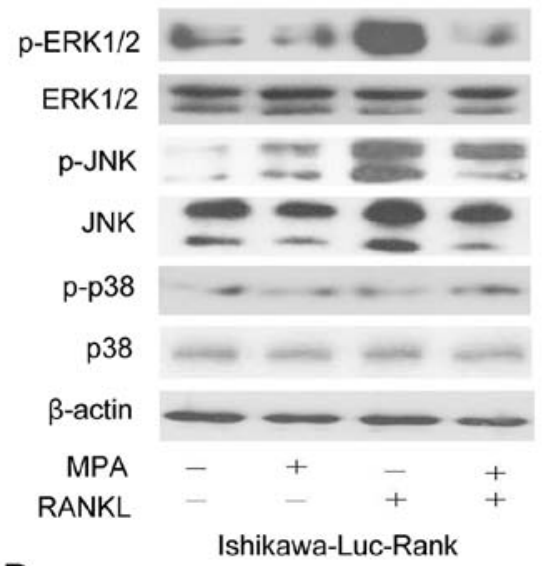

D

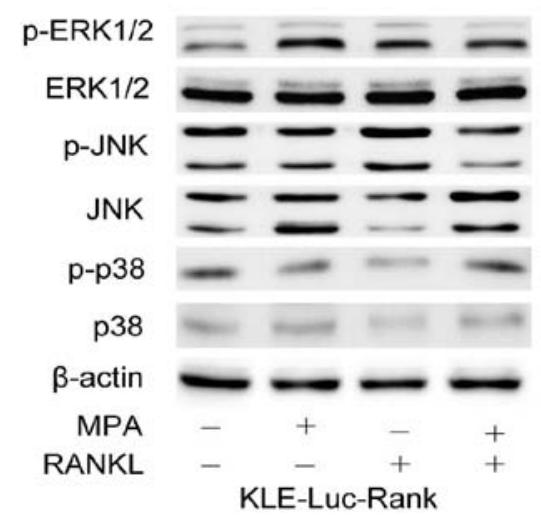

Figure 5. RANKL binding to RANK activates the MAPK and NF- $\kappa$ B pathways. (A) RANKL induced the phosphorylation of ERK1/2 and JNK in IshikawaLuc-Rank cells and had no obvious effect on p38. The cells were stimulated with $1 \mu \mathrm{g} / \mathrm{ml}$ RANKL for the indicated time periods. The total ERK1/2, JNK, p38 and $\beta$-actin protein levels are shown. (B) The activated form of NF- $\kappa$ B2 protein (p52) was detected in the cell nucleus. (C and D) MPA, RANKL and MPA + RANKL stimulated Ishikawa-Luc-Rank and KLE-Luc-Rank cells. The MAPK pathway was activated by RANKL only, whereas MPA inhibited the activation of the MAPK pathway induced by RANKL.

(Fig. 6C). These data revealed that MPA efficiently inhibited endometrial tumor growth in vivo, and MPA could also impede the oncogenic role of RANKL. Tumor tissues were embedded in paraffin and stained with hematoxylin and eosin (H\&E). The expression levels of RANK, RANKL and Ki-67 were determined by immunohistological assay (Fig. 6D). In the MPA- and MPA + RANKL-treated groups, lower expression of Ki-67 was consistent with smaller tumor volumes.

\section{Discussion}

RANKL is regarded as a promising therapy strategy for malignant tumors (6), and agents that block RANKL to exacerbate antitumor effect have raised considerable concern (15). Here, we revealed that RANK/RANKL expression was significantly higher in EC tissue than in normal endometrium and the expression of RANK/RANKL was positively correlated with myometrial invasion, lymph node metastasis, and lymphovascular space involvement, suggesting that targeting of RANK/RANKL could be a therapy for aggressive EC. Meanwhile, specific overexpression of RANK in Ishikawa and KLE cells markedly increased the proliferation, migration and invasion in vitro. Furthermore, MPA efficiently inhibited the tumorigenicity in nude mice in vivo. Therefore, we conclude that RANK/RANKL expression may regulate EC-associated metastatic behavior.

The expression of PRB is essential to progesterone action. Dai et al (16) demonstrated that PR expression, particularly the expression of PRB, appeared to be imperative for progesterone reaction, since the inhibitory actions of progesterone on cell proliferation and invasiveness may be due mainly to PRB activity. KLE has been identified as a poorly differentiated EC cell line that lacks PRB expression (17). Ai et al (18) observed that MPA inhibited the proliferation of Ishikawa cells, whereas it promoted the growth of KLE cells due to their divergent PRB statuses. KLE is regarded as an aggressive type 2 EC cell and progesterone-resistant EC cell (hormone receptor-negative). Therefore, we used Ishikawa (high levels of PRB) and KLE (low levels of PRB), which both have low levels of RANK. Our data confirmed that the effect of MPA-mediated PRB effectively inhibited the proliferation, migration and invasion of the RANKL-induced IshikawaLuc-Rank cells in vitro, whereas there was no change in the KLE-Luc-Rank cells. Thus, RANKL may be an important therapeutic target for the treatment of progesterone-resistant and aggressive EC. 


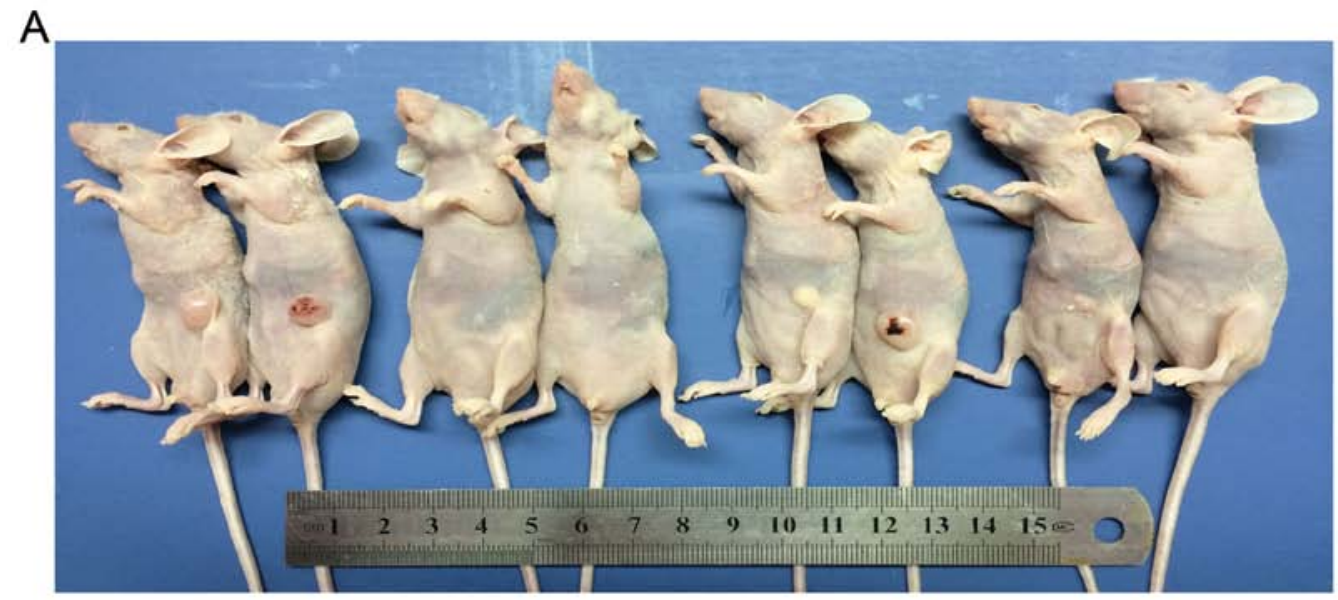

B

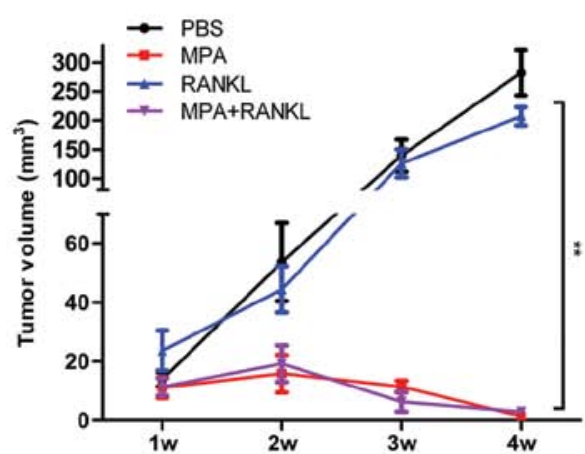

C

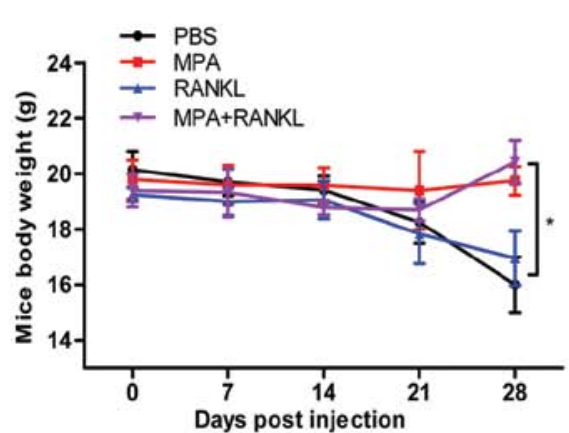

D

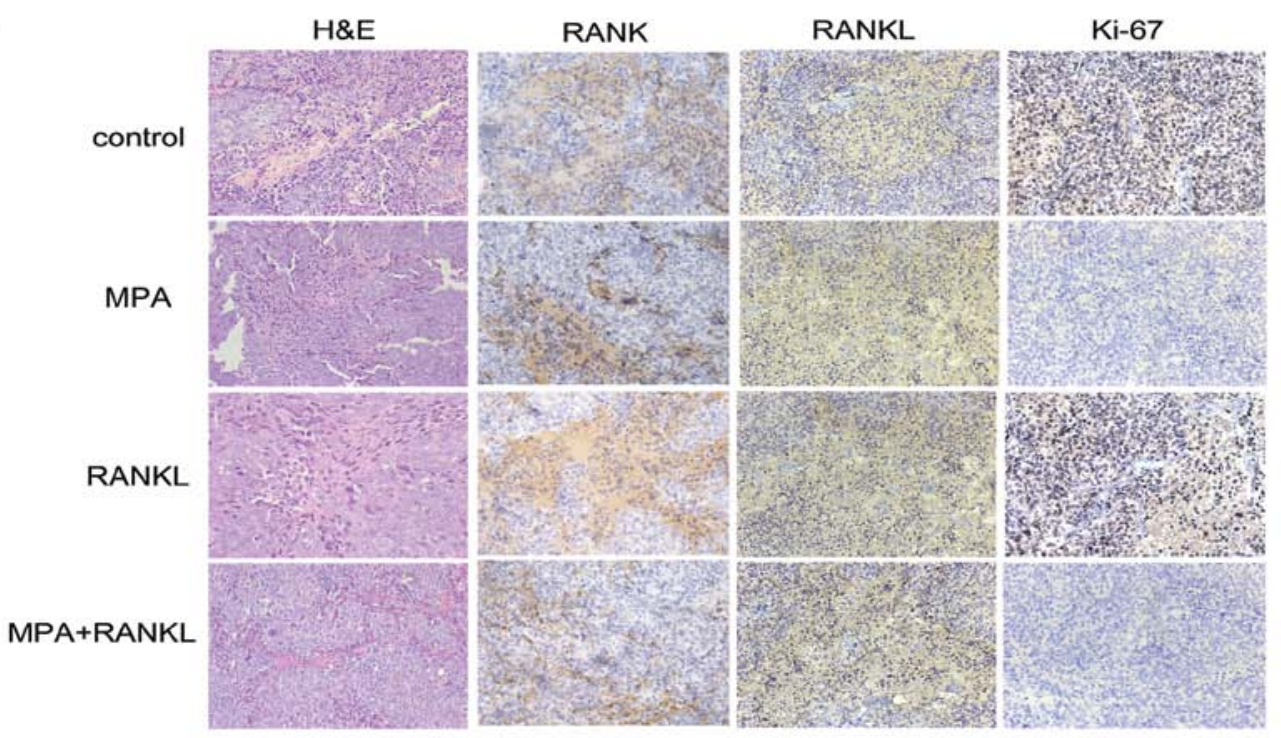

Figure 6. Tumorigenicity assay in nude mice. (A) Tumor-bearing mice at the study end-point. (B) Tumor volume was calculated as length $\mathrm{x}$ width ${ }^{2} \mathrm{x} 0.5$. Results represent the mean sizes of the xenografted tumors. (C) The weights of mice were recorded during the period of tumorigenicity. Results indicate the mean weight. ${ }^{*} \mathrm{P}<0.05,{ }^{* *} \mathrm{P}<0.01$. (D) Staining with hematoxylin and eosin $(\mathrm{H} \& \mathrm{E})$ or immunohistochemical staining for RANK, RANKL and Ki-67 in mouse tumor tissues.

The present study provides the initial evidence of the expression and function of RANK/RANKL in EC. It was previously proven that tumor cells that express RANK could activate the RANK-RANKL pathway (19-21). Considering the extensive regulatory role of RANK/RANKL, we constructed a cell-based model of high RANK expression. In the present study, we demonstrated that the stimulation of EC cells by
RANKL activated specific downstream pathways, including the ERK1/2, JNK and NF- $\kappa$ B pathways. In addition, we explored the interaction between RANK/RANKL and $\mathrm{PRB} / \mathrm{MPA}$ treatment in terms of the biological behavior of EC cells in vitro and in vivo.

Recent studies have highlighted that RANKL is upregulated in a broad range of tumors and bone-related metastases, 
mostly through the parathyroid hormone-related protein, which is produced by hormones, cytokines and the tumor cells themselves (22). The binding of RANKL to RANK causes receptor trimerization and initiates a cascade of reactions that facilitates the differentiation and maturity of tumor cells (13). This action leads to the enrollment of various molecules, including TNF receptor-associated factor (TRAF)-6, which activates the MAPK pathway. The activation of MAPK signaling cascades exerts a crucial role in transmitting extracellular signals to regulate a variety of cellular responses implicated in proliferation, differentiation, migration and apoptosis (23-25). These studies provide new insights into the critical cellular roles of RANKL in the development and progression of cancer.

Activated MAP kinases which transform extracellular stimuli into physiological responses phosphorylate molecules, including cytoskeletal proteins and mitochondrial proteins, and also regulate a wide range of transcription factors. NF- $\kappa \mathrm{B}$ proteins, which belong to the transcription factor superfamily, are involved in preventing cells from undergoing apoptosis inducing by DNA variation or cytokine treatment (26). Stimulation of the $N F-\kappa B$ pathway can activate a series of downstream molecules and in turn lead to their ubiquitination and degradation. These reactions can trigger the translocation of $\mathrm{NF}-\kappa \mathrm{B}$ proteins and initiate the expression of specific cellular genes. Accumulating evidence supports that $\mathrm{NF}-\kappa \mathrm{B}$ proteins are associated with cellular proliferation and apoptosis, which can be activated through the MAPK pathway $(27,28)$. We demonstrated that NF- $\kappa \mathrm{B}$ signaling mediated the apoptosis in EC cells induced by MPA, since the activated form of NF- $\kappa$ B2 protein (p52) was detected in the cell nucleus.

OPG plays a role in blocking the interaction between RANK and RANKL. We also demonstrated that the serum levels of OPG in EC patients were lower than those in healthy controls, whereas the levels of RANKL were higher. The reduction of serum levels of OPG may be related to an enhancement of the interaction between RANK and RANKL, which could activate the MAPK pathways to promote tumor progression.

Based on the results discussed above, it is clearly evident that RANK/RANKL is involved in the development and progression of EC, providing new perspectives in the analysis of the biological mechanisms of EC. MPA/PRB administration plays a vitally important role in the inhibition of the proliferation, migration, and invasion of EC cells. Drug targeting of RANKL may be an appealing candidate for progesteroneresistant and aggressive EC. Further studies are warranted to investigate the applications and implications in future clinical work and research.

\section{Acknowledgements}

The present study was supported by the National Natural Science Foundation of China (nos. 81172477 and 81072140), the Project of the Science and Technology Commission of Shanghai Municipality (nos. 11ZR1440800 and 13JC1401303), and the Project of Outstanding Subject Leaders of the Shanghai Health System (no. XBR2013097).

\section{References}

1. Siegel R, Ma J, Zou Z and Jemal A: Cancer statistics, 2014. CA Cancer J Clin 64: 9-29, 2014.
2. Di Cristofano A and Ellenson LH: Endometrial carcinima. Annu Rev Pathol 2: 57-85, 2007.

3. Salvesen HB, Haldorsen IS and Trovik J: Markers for individualised therapy in endometrial carcinoma. Lancet Oncol 13: e353-e361, 2012.

4. Kong YY, Yoshida H, Sarosi I, Tan HL, Timms E, Capparelli C, Morony S, Oliveira-dos-Santos AJ, Van G, Itie A, et al: OPGL is a key regulator of osteoclastogenesis, lymphocyte development and lymph-node organogenesis. Nature 397: 315-323,1999.

5. Fata JE, Kong YY, Li J, Sasaki T, Irie-Sasaki J, Moorehead RA, Elliott R, Scully S, Voura EB, Lacey DL, et al: The osteoclast differentiation factor osteoprotegerin-ligand is essential for mammary gland development. Cell 103: 41-50, 2000.

6. Beleut M, Rajaram RD, Caikovski M, Ayyanan A, Germano D, Choi Y, Schneider P and Brisken C: Two distinct mechanisms underlie progesterone-induced proliferation in the mammary gland. Proc Natl Acad Sci USA 107: 2989-2994, 2010.

7. Armstrong AP, Tometsko ME, Glaccum M, Sutherland CL, Cosman D and Dougall WC: A RANK/TRAF6-dependent signal transduction pathway is essential for osteoclast cytoskeletal organization and resorptive function. J Biol Chem 277: 44347-44356, 2002.

8. Darnay BG, Haridas V, Ni J, Moore PA and Aggarwal BB: Characterization of the intracellular domain of receptor activator of NF-kappaB (RANK). Interaction with tumor necrosis factor receptor-associated factors and activation of NF-kappaB and c-Jun N-terminal kinase. J Biol Chem 273: 20551-20555, 1998.

9. Mori K, Le Goff B, Berreur M, Riet A, Moreau A, Blanchard F, Chevalier C, Guisle-Marsollier I, Léger J, Guicheux J, et al: Human osteosarcoma cells express functional receptor activator of nuclear factor-kappa B. J Pathol 211: 555-562, 2007.

10. Zhang L, Teng Y, Zhang Y, Liu J, Xu L, Qu J, Hou K, Yang X, Liu Y and Qu X: C-Src-mediated RANKL-induced breast cancer cell migration by activation of the ERK and Akt pathway. Oncol Lett 3: 395-400, 2012.

11. Gonzalez-Suarez E, Jacob AP, Jones J, Miller R Roudier-Meyer MP, Erwert R, Pinkas J, Branstetter D and Dougall WC: RANK ligand mediates progestin-induced mammary epithelial proliferation and carcinogenesis. Nature 468: 103-107, 2010.

12. Schramek D, Leibbrandt A, Sigl V, Kenner L, Pospisilik JA, Lee HJ, Hanada R, Joshi PA, Aliprantis A, Glimcher L, et al: Osteoclast differentiation factor RANKL controls development of progestin-driven mammary cancer. Nature 468: 98-102, 2010.

13. Palafox M, Ferrer I, Pellegrini P, Vila S, Hernandez-Ortega S, Urruticoechea A, Climent F, Soler MT, Muñoz P, Viñals F, et al: RANK induces epithelial-mesenchymal transition and stemness in human mammary epithelial cells and promotes tumorigenesis and metastasis. Cancer Res 72: 2879-2888, 2012.

14. Schramek D, Sigl V and Penninger JM: RANKL and RANK in sex hormone-induced breast cancer and breast cancer metastasis. Trends Endocrinol Metab 22: 188-194, 2011.

15. Santini D, Schiavon G, Vincenzi B, Gaeta L, Pantano F, Russo A, Ortega C, Porta C, Galluzzo S, Armento G, et al: Receptor activator of NF-kB (RANK) expression in primary tumors associates with bone metastasis occurrence in breast cancer patients. PLoS One 6: e19234, 2011.

16. Dai D, Wolf DM, Litman ES, White MJ and Leslie KK: Progesterone inhibits human endometrial cancer cell growth and invasiveness: down-regulation of cellular adhesion molecules through progesterone B receptors. Cancer Res 62: 881-886, 2002.

17. Leslie KK, Kumar NS, Richer J, Owen G, Takimoto G, Horwitz KB and Lange C: Differential expression of the A and B isoforms of progesterone receptor in human endometrial cancer cells. Only progesterone receptor B is induced by estrogen and associated with strong transcriptional activation. Ann NY Acad Sci 828: 17-26, 1997.

18. Ai ZH, Wang J, Wang YD, Lu LH, Tong JQ and Teng YC: Overexpressed epidermal growth factor receptor (EGFR)induced progestin insensitivity in human endometrial carcinoma cells by the EGFR/mitogen-activated protein kinase signaling pathway. Cancer 116: 3603-3613, 2010.

19. Jones DH, Nakashima T, Sanchez OH, Kozieradzki I, Komarova SV, Sarosi I, Morony S, Rubin E, Sarao R, Hojilla CV, et al: Regulation of cancer cell migration and bone metastasis by RANKL. Nature 440: 692-696, 2006.

20. Armstrong AP, Miller RE, Jones JC, Zhang J, Keller ET and Dougall WC: RANKL acts directly on RANK-expressing prostate tumor cells and mediates migration and expression of tumor metastasis genes. Prostate 68: 92-104, 2008. 
21. Mori K, Le Goff B, Charrier C, Battaglia S, Heymann D and Rédini F: DU145 human prostate cancer cells express functional receptor activator of NFkappaB: new insights in the prostate cancer bone metastasis process. Bone 40: 981-990, 2007.

22. Wittrant Y, Théoleyre S, Chipoy C, Padrines M, Blanchard F, Heymann D and Rédini F: RANKL/RANK/OPG: new therapeutic targets in bone tumours and associated osteolysis. Biochim Biophys Acta 1704: 49-57, 2004.

23. Nebreda AR and Porras A: p38 MAP kinases: beyond the stress response. Trends Biochem Sci 25: 257-260, 2000.

24. Schieven GL: The biology of p38 kinase: a central role in inflammation. Curr Top Med Chem 5: 921-928, 2005.

25. Manning AM and Davis RJ: Targeting JNK for therapeutic benefit: from junk togold? Nat Rev Drug Discov 2: 554-565, 2003.
26. Yamamoto $\mathrm{Y}$ and Gaynor RB: Therapeutic potential of inhibition of the NF-kappaB pathway in the treatment of inflammation and cancer. J Clin Invest 107: 135-142, 2001.

27. Barkett $\mathrm{M}$ and Gilmore TD: Control of apoptosis by Rel/ NFkappaB transcription factors. Oncogene 18: 6910-6924, 1999.

28. Hashimoto I, Koizumi K, Tatematsu M, Minami T, Cho S Takeno N, Nakashima A, Sakurai H, Saito S, Tsukada K and Saiki I: Blocking on the CXCR4/mTOR signaling pathway induces the anti-metastatic properties and autophagic cell death in peritoneal disseminated gastric cancer cells. Eur J Cancer 44: 1022-1029, 2008. 